\title{
Development and validation of a prognostic nomogram based on the log odds of positive Iymph nodes (LODDS) for breast cancer
}

\author{
Jiahuai Wen ${ }^{1, *}$, Feng Ye ${ }^{1, *}$, Xiaofang He ${ }^{1}$, Shuaijie Li $^{1}$, Xiaojia Huang ${ }^{1}$, Xiangsheng \\ $\mathrm{Xiao}^{1}$ and Xiaoming $\mathrm{Xie}^{1}$ \\ ${ }^{1}$ Department of Breast Oncology, Sun Yat-Sen University Cancer Center, State Key Laboratory of Oncology in South China, \\ Collaborative Innovation Center for Cancer Medicine, Guangzhou, Guangdong, China \\ * These authors have contributed equally to this work
}

Correspondence to: Xiaoming Xie, email: xiexm@sysucc.org.cn

Keywords: breast cancer, LODDS, prognosis, nomogram, surgery

Received: November 23, 2015 Accepted: February 24, $2016 \quad$ Published: March 15, 2016

\section{ABSTRACT}

Background: To evaluate the prognostic effect of log odds of positive lymph nodes (LODDS) and develop a nomogram for survival prediction in breast cancer patients at the time of surgery.

Results: LODDS was an independent risk factor for cancer-related death in breast cancer (hazard ratio: 1.582, 95\%CI: 1.190-2.104). Menopausal status, tumor size, pathological lymph node staging, estrogen receptor status and human epidermal growth factor receptor-2 status were also included in the nomogram. The calibration plots indicated optimal agreement between the nomogram prediction and actual observation. Discrimination of nomogram was superior to the seventh edition TNM staging system [C-index: 0.745 vs. $0.721(p=0.03)$ in training cohort; 0.796 vs. 0.726 $(p<0.01)$ in validation cohort].

Methods: We retrospectively evaluated 2023 breast cancer patients from Jan 2002 to Dec 2008 at our center. The cohort was randomly divided into training cohort and validation cohort. Univariate and multivariate analyses were performed to identify prognostic factors, and nomogram was established using Cox regression model in training cohort. External validation of the nomogram was performed in the validation cohort.

Conclusions: The LODDS is an independent prognostic indicator in breast cancer and the novel nomogram can provide individual prediction of cancer-specific survival and help prognostic assessment for breast cancer patients.

\section{INTRODUCTION}

Breast cancer is the most common cancer and the leading cause of cancer death in women worldwide. According to the National Cancer Institute, more than 230,000 new cases and 40,000 deaths were reported in 2014 in the United States [1]. Although the advances in treatments of breast cancer have improved the therapeutic effect [2], local recurrences and distant metastases remain the main challenges to clinicians [3].

In breast cancer, axillary lymph nodes (LNs) assessment is crucial for the evaluation of disease severity, treatment options and prognosis assessment [4]. Increase in the number of positive LNs is independently related to increased risk of recurrence and decrease of overall survival $[5,6]$. Traditionally, the National Comprehensive Cancer Network (NCCN) Guidelines recommend the number of positive LNs for axillary assessment and classify patients into different stages according to the American Joint Committee on Cancer (AJCC) tumornode-metastasis (TNM) staging system. However, the TNM staging system does not consider the total number of LNs retrieved, and limited total LNs may cause stage migration and influence the accuracy of prognosis assessment $[7,8]$.

Ratio-based nodal systems were proposed as alternative tools of LN assessment in recent studies. Log odds of positive lymph nodes (LODDS), defined as the log of odds between number of positive nodes and number of negative nodes, showed superiority over the AJCC $\mathrm{pN}$ staging in several cancers, like gastric cancer 
[9], pancreatic cancer [10] and colorectal cancer [11, 12], especially when insufficient lymph nodes were retrieved. In breast cancer, the LODDS was considered as an independent prognostic factor and more reliable than the $\mathrm{pN}$ staging $[13,14]$.

Nomogram is graphical calculating tool used to quantify risk through intuitive graphs. It could provide individualized prognostic information based on the prognostic factors and be more accurate than the conventional staging systems for predicting prognosis in some cancers $[15,16]$.

In this study, we aimed to assess the prognostic significance of the LODDS and compare its prognostic value with $\mathrm{pN}$ staging in breast cancer. Additionally, a predictive postoperative nomogram based on the LODDS was developed and externally validated in breast cancer patients underwent modified radical mastectomy.

\section{RESULTS}

\section{Baseline characteristics}

A total of 2023 female patients with primary nonmetastatic invasive breast cancer were enrolled. Then, 1494 patients were randomly selected as training cohort and 519 patients were included in the validation cohort. Patient characteristics were listed in Table 1. The mean age of the overall cohort was 49.4 years old (range 2286 years), and $171(8.5 \%)$ patients were under the age of 35 . There were 1077 (53.2\%) patients suffered regional lymph node metastases, and 305 (15.1\%) patients had less than 10 total lymph nodes retrieved. Stage I, II and III accounted for $26.6 \%, 52.7 \%$ and $20.7 \%$ of the study cohort, respectively. Luminal subtype comprised $76.6 \%$ of total participants, and $218(10.8 \%)$ and $254(12.6 \%)$ were HER2 over-expressing subtype and triple-negative subtype respectively. No significant difference was observed between the training cohort and validation cohort regarding the clinicopathological factors analyzed.

\section{Analysis of the prognostic impact of LODDS}

The mean LODDS was -0.878 (range: -1.88 to $1.79)$ in the training cohort. Cancer-related death occurred in $336(16.6 \%)$ patients during a median follow-up time of 75.1 (range 5-144) months after surgery. Table S1 listed the median survival time of different levels according to the value of LODDS with an interval of 0.5. Patients with similar prognosis were classified into four groups: LODDS1 $($ LODDS $\leq-1.00)$, LODDS2 $(-1.00<$ LODDS $\leq$ $0)$, LODDS3 $(0<$ LODDS $\leq 1.5)$ and LODDS4 (LODDS $>1.5$ ). The 10-year survival rates of patients in LODDS1, LODDS2, LODDS3 and LODDS4 were $87.1 \%, 74.0 \%$, $48.2 \%$ and $14.4 \%$ respectively $(p<0.001)$.
In univariate analysis, age, menopausal status, histologic grade, tumor size, hormone receptor statuses, HER2 status, pN stages and LODDS were associated with cancer-specific survival of breast cancer patients (Table 2 , all $p<0.05$ ). In the step 1 and step 2 multivariate survival analyses, $\mathrm{pN}$ stages and LODDS were identified as independent prognostic factors respectively. In the step 3 multivariate survival analysis, $\mathrm{pN}$ stages and LODDS remained statistically significant in the same model (Table 2 , all $p<0.05$ ). Other independent prognostic factors included menopausal status, tumor size, ER status and HER2 status.

Subgroup analysis indicated that $\mathrm{pN}$ stages and LODDS were both independently associated with the prognosis of breast cancer in both the retrieved LNs $<10$ and $\geq 10$ subgroups (Table 3 , all $p<0.05$ ). The corresponding AUCs for $\mathrm{pN}$ and LODDS were 0.715 (95\% CI: 0.683-0.746) and 0.709 (95\% CI: 0.694-0.734) respectively, indicating that $\mathrm{pN}$ stages may have better discrimination capability than the LODDS, although the difference was not significant $(p=0.408)$. PN stages manifested superior model fitness over the LODDS with smaller -2LLR and AIC. In the subgroup of more than 10 lymph nodes-retrieved, the $\mathrm{pN}$ stages remained superior model fitness. However, the LODDS fitted the model better than the $\mathrm{pN}$ stages when limited lymph nodes were obtained (Table 3)

\section{Prognostic nomogram for CSS}

A nomogram based on the results of step 3 multivariate survival analysis was established (Figure 1). By summing the scores and locating on the total score scale, the estimated probability of cancer-specific survival at 5-year and 10-year could be determined.

In the training cohort, the $\mathrm{C}$-index for the established nomogram was 0.745 (95\% CI: 0.721 to 0.769$)$ and higher than that for the TNM staging system $(0.721 ; 95 \%$ CI: 0.692 to $0.753, p=0.03$ ). In the validation cohort, the C-index for the nomogram $(0.796 ; 95 \%$ CI: 0.756 to 0.860 ) was also higher than that for the TNM staging system $(0.726 ; 95 \%$ CI: 0.665 to $0.787, p<0.01)$. The calibration plots indicated an optimal agreement between the nomogram prediction and actual observation for 5-year and 10-year CSS in both the training cohort and validation cohort (Figure 2). After sorting by total score of nomogram, patients were classified evenly into four subgroups (total points: 0 to $30,30.1$ to $77,77.1$ to $115,115.1$ to 300$)$. Each subgroup experienced distinct prognosis in both training cohort and validation cohort ( $p<0.05$, Figure 3 ). Taking the group of score $0-30$ as reference, the HR for sequential subgroups were 3.105 , 4.611 and 10.686 in training cohort and 3.184, 7.201 and 17.212 in the validation cohort respectively. 
Table 1: Clinicopathological characteristics of patients in the training and validation cohort

\begin{tabular}{|c|c|c|c|c|}
\hline Characteristic & $\begin{array}{l}\text { All patients } \\
(n=\mathbf{2 0 2 3})\end{array}$ & $\begin{array}{l}\text { Training cohort } \\
(n=1504)\end{array}$ & $\begin{array}{l}\text { Validation cohort } \\
(n=519)\end{array}$ & $p$ value \\
\hline Age & & & & 0.836 \\
\hline$<35$ & $171(8.5)$ & $126(8.4)$ & $45(8.7)$ & \\
\hline$>35$ & $1852(91.5)$ & $1378(91.6)$ & $474(91.3)$ & \\
\hline Menopause & & & & 0.457 \\
\hline Yes & $540(26.7)$ & $395(26.3)$ & $145(27.9)$ & \\
\hline No & $1483(73.3)$ & $1109(73.7)$ & $374(72.1)$ & \\
\hline Tumor type & & & & 0.630 \\
\hline IDC & 1963 (97.0) & 1461 (97.1) & $502(96.7)$ & \\
\hline ILC & $60(3.0)$ & $43(2.9)$ & $17(3.3)$ & \\
\hline Histologic grade & & & & 0.865 \\
\hline G1 & $76(3.7)$ & $56(3.6)$ & $20(3.8)$ & \\
\hline G2 & $1214(60.0)$ & $900(60.0)$ & $314(60.5)$ & \\
\hline G3 & $733(36.3)$ & $548(36.4)$ & $185(35.7)$ & \\
\hline Tumor size & & & & 0.947 \\
\hline $\mathrm{T} 1$ & $852(42.1)$ & $633(42.1)$ & \begin{tabular}{|l|}
$219(42.2)$ \\
\end{tabular} & \\
\hline $\mathrm{T} 2$ & $1068(52.8)$ & $793(52.7)$ & $275(53.0)$ & \\
\hline T3 & $103(5.1)$ & $78(5.2)$ & $25(4.8)$ & \\
\hline AJCC LN status & & & & 0.216 \\
\hline N0 & $946(46.8)$ & $712(47.3)$ & $234(45.1)$ & \\
\hline N1 & $553(27.3)$ & $411(27.3)$ & $142(27.4)$ & \\
\hline $\mathrm{N} 2$ & $308(15.2)$ & $215(14.3)$ & \begin{tabular}{|l|}
$93(17.9)$ \\
\end{tabular} & \\
\hline $\mathrm{N} 3$ & $216(11.2)$ & $166(11.0)$ & $50(9.6)$ & \\
\hline Total LN & & & & 0.455 \\
\hline$<<10$ & $305(15.1)$ & $232(15.4)$ & $73(14.1)$ & \\
\hline$\geq 10$ & $1718(84.9)$ & $1272(84.6)$ & $446(85.9)$ & \\
\hline$\overline{E R}$ & & & & 0.280 \\
\hline Positive & $1264(62.5)$ & $950(63.2)$ & $314(60.5)$ & \\
\hline Negative & $759(37.5)$ & $554(36.8)$ & $205(39.5)$ & \\
\hline PR & & & & 0.364 \\
\hline Positive & $1381(68.3)$ & $1035(68.8)$ & \begin{tabular}{|l|}
$346(66.7)$ \\
\end{tabular} & \\
\hline Negative & $642(31.7)$ & $469(31.2)$ & $173(33.3)$ & \\
\hline HER-2 & & & & 0.069 \\
\hline Positive & $550(27.2)$ & $393(26.1)$ & $157(30.3)$ & \\
\hline Negative & $1473(72.8)$ & $1111(73.9)$ & $362(69.7)$ & \\
\hline Cancer-specific survival & & & & 0.566 \\
\hline Alive & $1687(83.4)$ & $1250(83.1)$ & $437(84.2)$ & \\
\hline Death & $336(16.6)$ & $254(16.9)$ & $82(15.8)$ & \\
\hline LODDS (mean \pm SD) & $-0.878 \pm 0.715$ & $-0.879 \pm 0.721$ & $-0.875 \pm 0.696$ & 0.909 \\
\hline
\end{tabular}

Abbreviations: SD standard deviation, IDC Invasive ductal carcinoma, ILC Invasive lobular carcinoma, LN lymph node, ER Estrogen receptor, PR Progesterone receptor, HER2 Human epidermal growth factor receptor-2, LODDS log odds of positive lymph nodes

\section{DISCUSSION}

In the current study, we evaluated 2023 breast cancer patients who received modified radical mastectomy and the results shown that: (1) the LODDS was an independent prognostic parameter along with the AJCC pN staging system; (2) LODDS manifested better model fitness when inadequate lymph nodes $(<10)$ were obtained in the surgery; (3) a nomogram was established and performed well in predicting survival in breast cancer patients.

Axillary lymph node status is considered as the most important prognostic factor in breast cancer patients. In the AJCC TNM staging system, the $\mathrm{pN}$ stages are defined as N0 (no positive lymph node), N1 (1-3 lymph nodes), N2 (4-9 lymph nodes) and N3 ( $\geq 10$ lymph nodes), but the 
Table 2: Univariate and multivariate analyses on CSS in breast cancer patients

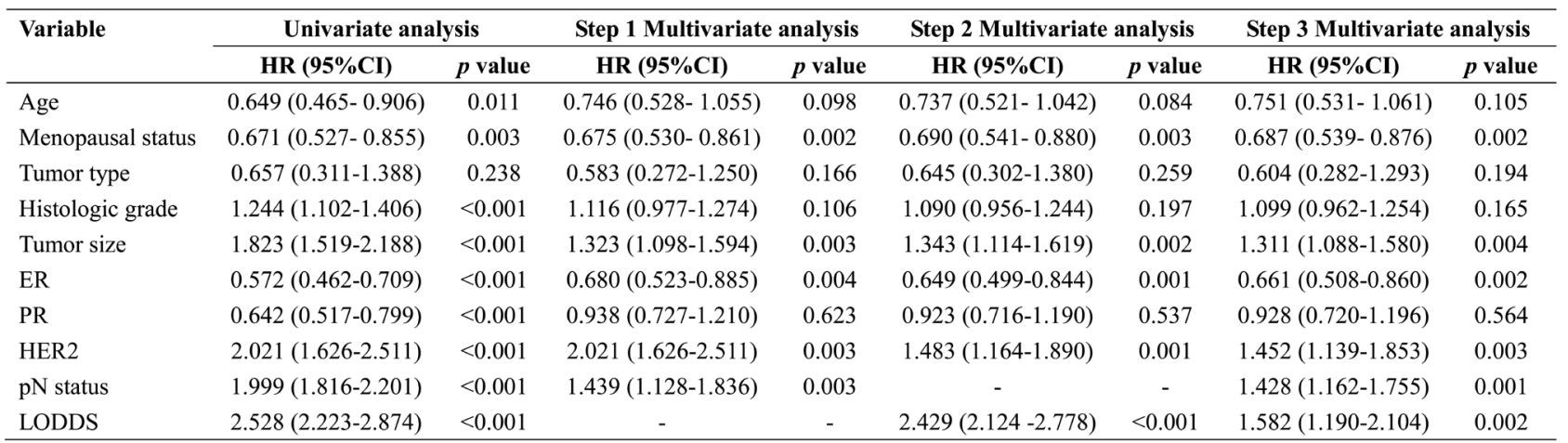

Abbreviations: CSS cancer-specific survival, HR hazard ratio, CI confidence interval, ER Estrogen receptor,PR Progesterone receptor, HER2 Human epidermal growth factor receptor- 2 , pN pathological lymph node, LODDS log odds of positive lymph nodes

Table 3: Subgroup multivariate analysis and prognostic performance of pN staging and LODDS.

\begin{tabular}{|l|l|l|l|l|l|}
\hline Lymph nodes retrieved & Category & HR (95\%CI) $^{\mathbf{a}}$ & $\boldsymbol{p}$ value & -2LLR & AIC \\
\hline \multirow{2}{*}{ All patients } & $\mathrm{pN}$ & $1.439(1.128-1.836)$ & $<0.001$ & 2352.45 & 4706.90 \\
\cline { 2 - 6 } & LODDS & $2.429(2.124-2.778)$ & $<0.001$ & 2356.10 & 4715.23 \\
\hline \multirow{2}{*}{ Lymph nodes $\geq 10$} & $\mathrm{pN}$ & $1.920(1.049-1.558)$ & $<0.001$ & 2048.12 & 4099.65 \\
\cline { 2 - 6 } & LODDS & $2.489(2.159-2.869)$ & $<0.001$ & 2051.37 & 4104.91 \\
\hline \multirow{2}{*}{ Lymph nodes $<10$} & $\mathrm{pN}$ & $1.777(1.156-2.734)$ & $<0.001$ & 189.94 & 381.88 \\
\cline { 2 - 6 } & LODDS & $2.018(1.293-3.150)$ & $<0.001$ & 188.78 & 379.56 \\
\hline
\end{tabular}

${ }^{a}$ Either lymph-nodes staging method was incorporated in the multivariate analysis respectively

Abbreviations: HR hazard ratio, CI confidence interval, LLR log-likelihood ratio, AIC Akaike's information criterion, $\mathrm{pN}$ pathological lymph node, LODDS log odds of positive lymph nodes.

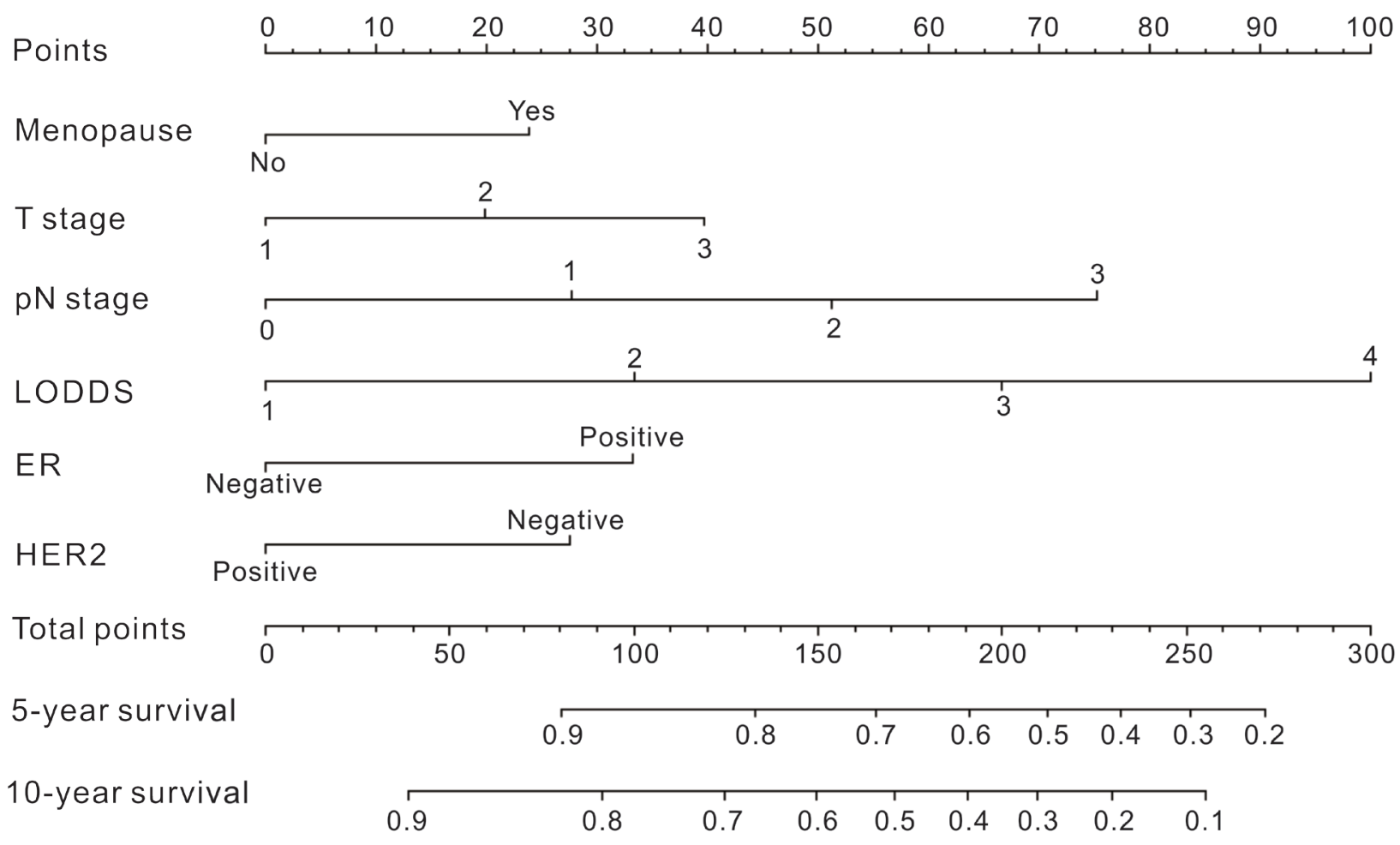

Figure 1: Nomogram for predicting the probability of cancer-specific survival in breast cancer patients. 
A

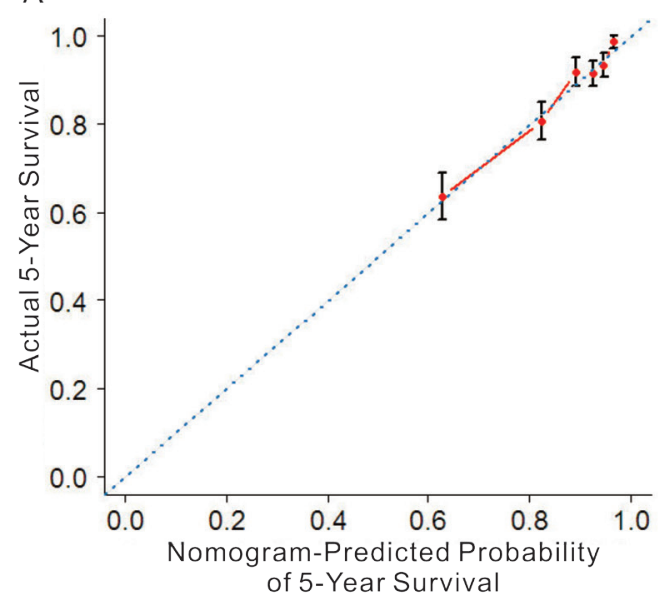

C

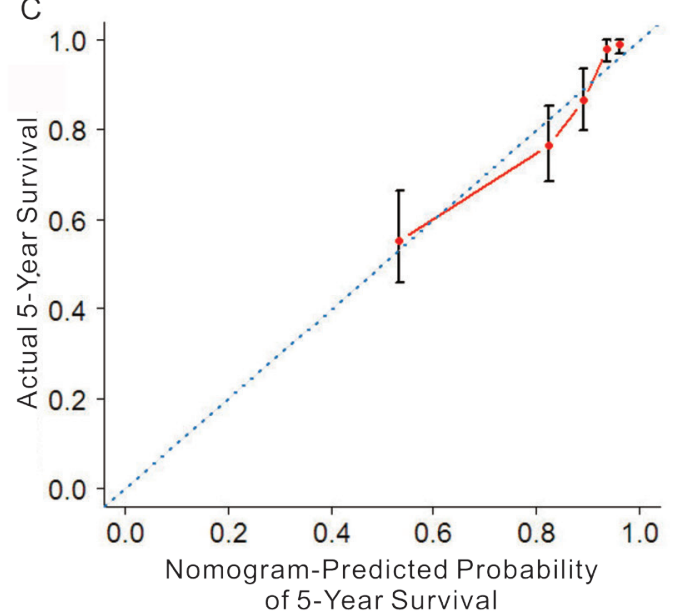

B

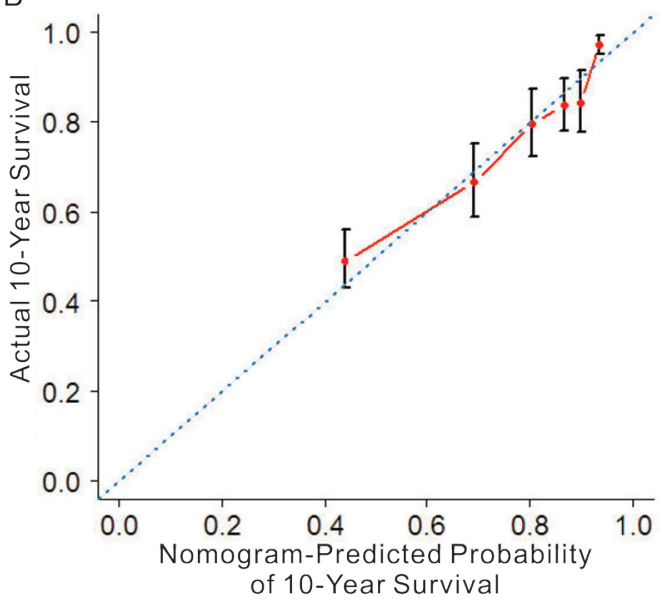

D

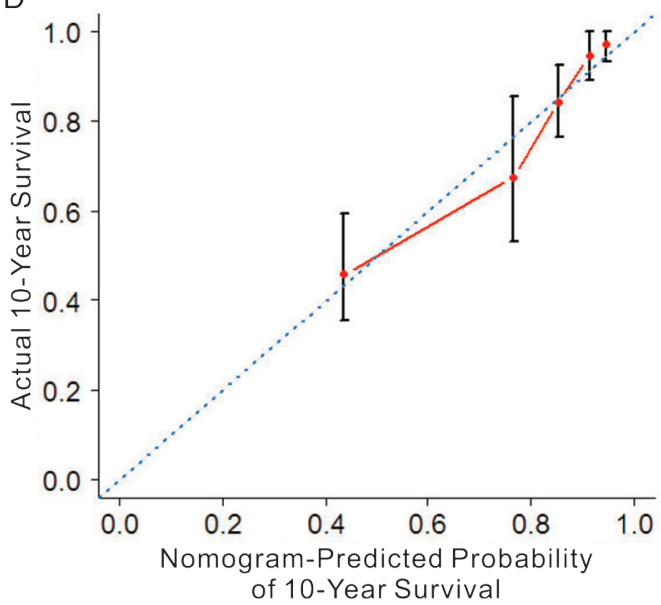

Figure 2: The calibration plots for predicting patient cancer-specific survival at each time point in the training cohort (A-B) and validation cohort (C-D). Nomogram-predicted CSS is plotted on the $\mathrm{x}$-axis; actual CSS is plotted on the y-axis. The $45^{\circ}$ reference line indicates the perfect predictions.
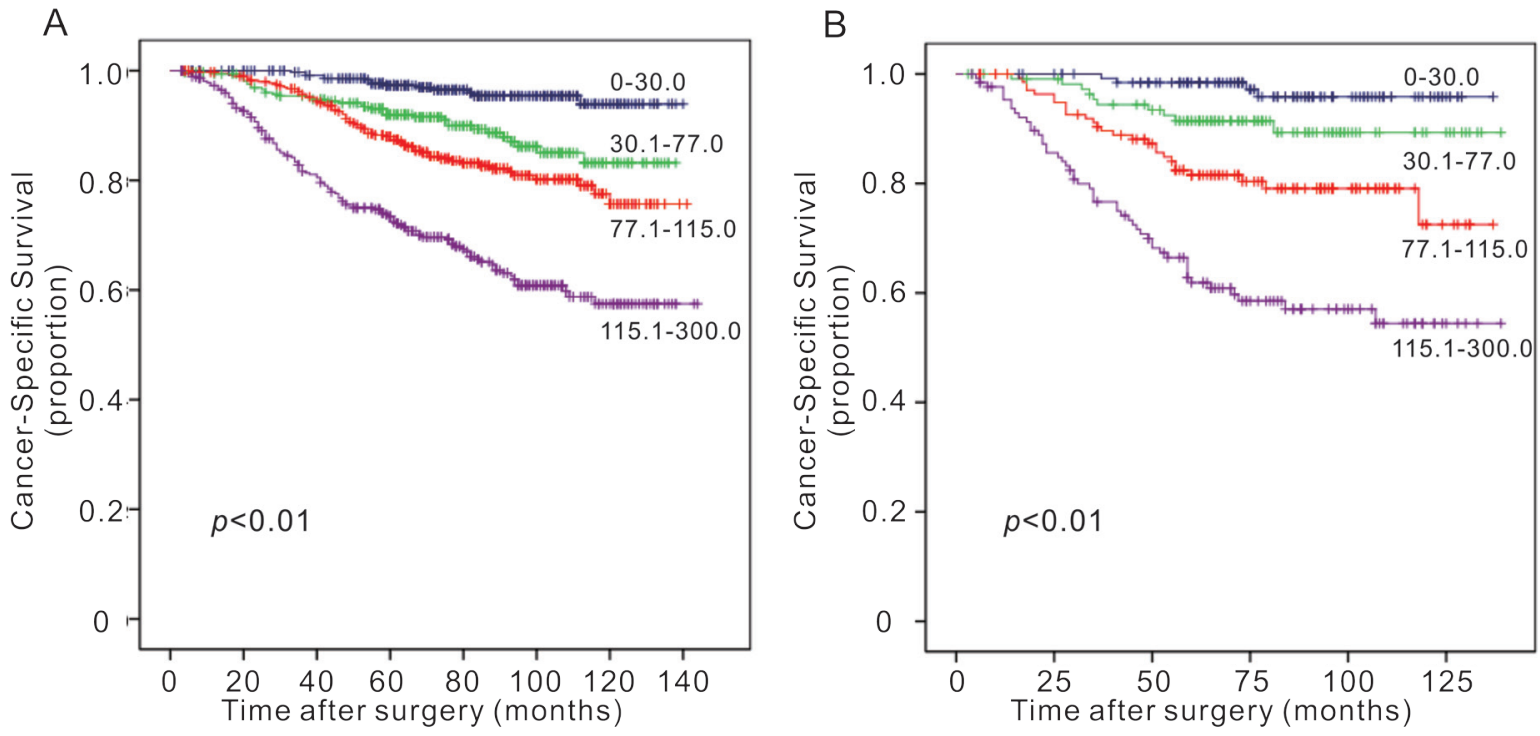

Figure 3: Survival curves of breast cancer patients in (A) training cohort and (B) validation cohort according to the nomogram-based classification. Participants were classified into four subgroups based on the nomogram score: 0- 0, 30.1-77, 77.1115 and 115.1- 300 . 
accuracy of $\mathrm{pN}$ staging may be lowered by the limited number of lymph nodes retrieved, called stage migration [17-19].

Previous studies had indicated that ratio-based LN staging systems are more accurate in predicting the prognosis when limited LNs are retrieved [20]. LODDS was reported to provide more accurate information of lymph node status and be superior to TNM staging systems. In the present study, survival rates between the LODDS 1 to 4 were significantly different $(p<0.05)$. When considering the total number of retrieved LNs, LODDS remained independently associated with the prognosis of breast cancer patients in the limited $(<10)$ and sufficient $(\geq 10$ ) retrieved-LNs groups respectively (both $p<0.05$ ). AIC and -2LLR were calculated to evaluate the model fitness, and LODDS only manifested better model fitness than $\mathrm{pN}$ staging in the limited retrieved-LNs group.

Since the LODDS is determined by the number of positive and negative LNs, more metastatic LNs indicate higher LODDS and $\mathrm{pN}$ staging. However, patient with same number of positive LNs may have various LODDS levels when considering the total number of retrieved LNs. Several studies have investigated the prognostic effect of negative LNs and proposed that negative LN should be considered when predicting the clinical outcomes in cancer patients $[22,23]$. Thus, the LODDS could improve the accuracy of LN assessment and better stratify patients with different prognosis when inadequate LNs were retrieved. On the other hand, LODDS may also underestimate the LN status when the ratio of positive and negative LNs is same. Thus we consider the LODDS as a complement to $\mathrm{pN}$ staging and could work together to further improve the accuracy of lymph node assessment in breast cancer patients.

Nomogram has been considered as an effective tool to quantify risks and maximize predictive accuracy in several cancer types [24-26]. The nomogram illustrated that the LODDS had major contribution to the prognosis of breast cancer patients, followed by $\mathrm{pN}$ stages. Although the $\mathrm{pN}$ staging system had a slightly better model fitness than the LODDS, the latter manifested higher hazard ratio, which was the possible reason for the largest impact on the nomogram. Calibration plots showed the optimal agreement of survival rates between the prediction and actual observation in the training cohort and validation cohort respectively, indicating the reliability of the established nomogram in the current study. Discrimination capability was assessed by the $\mathrm{C}$-index, and the nomogram showed better performance of prognosis prediction with higher C-index when compared with the AJCC TNM staging system.

There were several limitations in our study. Firstly, this was a single-center retrospective study and multicentric prospective studies are needed to reduce selection bias and prove the clinical value of the nomogram scoring system. Secondly, although the prediction accuracy of the nomogram scoring system exceeded that of the TNM staging system, there were $21.5 \%-25.5 \%$ of predictions made incorrectly. Tumorrelated markers could be incorporated into the nomogram for further improvement of the prediction accuracy.

In conclusion, our study demonstrated that LODDS was independently associated with the prognosis of breast cancer patients, and it performed well when limited lymph nodes were obtained. Furthermore, we established and validated a novel nomogram to predict the risk of cancerrelated death with better discrimination property than the traditional TNM staging system.

\section{MATERIALS AND METHODS}

\section{Study population}

Consecutive patients histologically diagnosed as breast cancer between January 2002 and December 2008 in Sun Yat-sen University Cancer Center (SYSUCC) were retrospectively reviewed. Inclusion criteria were as follows: (1) received modified radical mastectomy; (2) female; (3) pathological diagnosed as invasive ductal carcinoma (IDC) or invasive lobular carcinoma (ILC). Exclusion criteria included: (1) received neoadjuvant therapy before surgery; (2) had surgical treatment before admission; (3) with previous or coexisting cancers other than breast cancer; (4) confirmed metastasis; (5) tumor invaded to the chest wall or to the skin; (6) not enough data could be extracted. All patients were followed up to March 312015 or date of deaths. Every enrolled patient was randomly allocated as "training" or "validation" at the ratio of 3:1 and $75 \%$ of participants were selected as the training cohort. The remaining $25 \%$ of patients were grouped as the validation cohort.

\section{Clinical data collection}

Clinical characteristics collected for subsequent analysis included age, menstrual status, pathological diagnosis, histologic grade, tumor size, number of positive lymph nodes, number of total retrieved lymph nodes, hormone receptor and human epidermal growth factor receptor-2 (HER2) status and date of last followup or death. The clinical stages were classified according to the AJCC TNM staging system ( $7^{\text {th }}$ edition). The intrinsic subtypes were as follow: Luminal A (estrogen receptor $(\mathrm{ER})+$, progesterone receptor $(\mathrm{PR})+$, HER2 and Ki-67 $\leq 14 \%$ ), Luminal B (ER+ and HER2 + or Ki-67> 14\%), HER2 over-expressing (ER-, PR-, HER2+) and triple-negative breast cancer (ER-, PR-, HER2-). HER2 positive was defined as " $3+$ " in immunohistochemical test or "positive" in HER2 fluorescence in situ hybridization test. The follow-up of patients was started after the breast 
cancer surgery and performed through out-patient medical records, telephone or letters by Department of Follow-up $\&$ Medical Record Management.

\section{Statistical analyses}

Categorical data were described using numbers and percentages, and Chi square tests were performed to examine the differences between groups. LODDS was defined as $\log \frac{p L N+0.5}{t L N-p L N+0.5}$, where pLN is the number of positive lymph nodes and tLN is the total number of retrieved lymph nodes. 0.5 was added to both the numerator and denominator to avoid infinite singularity [27]. The primary endpoint assessed was cancer-specific survival (CSS), calculated from the time of pathological diagnosis to the date of cancer-related death or last follow-up. Intervals of LODDS in classification were determined by comparing median survival time according to LODDS with an interval of 0.5 and combining patients with similar prognosis. Univariate analysis and 3-steps multivariate analyses (Cox proportional hazards model) were performed to identify the independent variables associated with CSS. In step 1 and step 2 multivariate analyses, $\mathrm{pN}$ stages and LODDS status were included respectively, and both $\mathrm{pN}$ stages and LODDS status were included in step 3 multivariate analysis. Survival analyses were performed in the subgroups based on the number of retrieved lymph nodes ( $<10$ or $\geq 10$ ). Hazard ratios (HRs) and $95 \%$ confidence intervals (CIs) were calculated from the Cox regression model. Akaike's information criterion (AIC) and -2 log-likelihood ratio (-2LLR) within the Cox regression model were calculated to compare the model fitness between different lymph node staging systems.

A nomogram was developed based on the results of multivariate analysis. Backward step-down process was performed with AIC as a stopping rule [28]. 1,000 bootstrap resamples were used for internal validation of the training cohort and the external validation was performed by applying the nomogram to the validation cohort. Concordance index (C-index) was calculated for the evaluation of the performance of nomogram and the comparison with AJCC staging system. Calibration of the nomogram was performed by comparing the predicted survival with the observed survival in both the training cohort and validation cohort. SPSS (version 19.0, Chicago, IL, USA) and R software (version 3.0.1) with the survival and rms package were used for statistical analysis. A two-tailed $p$ value $<0.05$ was considered statistically significant.

\section{CONFLICTS OF INTEREST}

The authors declare that they have no conflict of interest.

\section{GRANT SUPPORT}

This work was supported by funds from the National Natural Science Foundation of China (81472575, 81272514); the Key Programme of the National Natural Science Foundation of China (31030061); the Science and Technology Planning Project of Guangdong and Guangzhou (2013B060300009, 2014J4100169).

\section{Ethics statement}

The study protocol was approved the by independent ethical committee/institutional review board of Sun Yatsen University Cancer Center (SYSUCC), and written informed consent about the scientific research was obtained from each participant prior to surgery. Patient records were anonymized and de-identified prior to analysis.

\section{REFERENCES}

1. Siegel RL, Miller KD and Jemal A. Cancer statistics, 2015. CA Cancer J Clin. 2015; 65:5-29.

2. Fedele P, Orlando L, Schiavone P, Calvani N, Caliolo C, Quaranta A, Nacci A and Cinieri S. Recent advances in the treatment of hormone receptor positive HER2 negative metastatic breast cancer. Crit Rev Oncol Hematol. 2015; 94:291-301.

3. Carlson RW, Allred DC, Anderson BO, Burstein HJ, Edge SB, Farrar WB, Forero A, Giordano SH, Goldstein LJ, Gradishar WJ, Hayes DF, Hudis CA, Isakoff SJ, Ljung BM, Mankoff DA and Marcom PK, et al. Metastatic breast cancer, version 1.2012: featured updates to the NCCN guidelines. J Natl Compr Canc Netw. 2012; 10:821-829.

4. Fitzgibbons PL, Page DL, Weaver D, Thor AD, Allred DC, Clark GM, Ruby SG, O’Malley F, Simpson JF, Connolly JL, Hayes DF, Edge SB, Lichter A and Schnitt SJ. Prognostic factors in breast cancer. College of American Pathologists Consensus Statement 1999. Arch Pathol Lab Med. 2000; 124:966-978.

5. Axelsson CK, Mouridsen HT and Zedeler K. Axillary dissection of level I and II lymph nodes is important in breast cancer classification. The Danish Breast Cancer Cooperative Group (DBCG). Eur J Cancer. 1992; 28A:1415-1418.

6. Recht A, Gray R, Davidson NE, Fowble BL, Solin LJ, Cummings FJ, Falkson G, Falkson HC, Taylor ST and Tormey DC. Locoregional failure 10 years after mastectomy and adjuvant chemotherapy with or without tamoxifen without irradiation: experience of the Eastern Cooperative Oncology Group. J Clin Oncol. 1999; 17:16891700.

7. Erbes T, Orlowska-Volk M, Zur HA, Rucker G, Mayer S, Voigt M, Farthmann J, Iborra S, Hirschfeld M, Meyer PT, 
Gitsch G and Stickeler E. Neoadjuvant chemotherapy in breast cancer significantly reduces number of yielded lymph nodes by axillary dissection. BMC Cancer. 2014; 14:4.

8. Schaapveld M, de Vries EG, van der Graaf WT, Otter R, de Vries J and Willemse PH. The prognostic effect of the number of histologically examined axillary lymph nodes in breast cancer: stage migration or age association? ANN Surg Oncol. 2006; 13:465-474.

9. Sun Z, Xu Y, Li DM, Wang ZN, Zhu GL, Huang BJ, Li $\mathrm{K}$ and $\mathrm{Xu} \mathrm{HM}$. Log odds of positive lymph nodes: a novel prognostic indicator superior to the number-based and the ratio-based $\mathrm{N}$ category for gastric cancer patients with $\mathrm{R} 0$ resection. Cancer-AM Cancer SOC. 2010; 116:2571-2580.

10. La Torre M, Nigri G, Petrucciani N, Cavallini M, Aurello P, Cosenza G, Balducci G, Ziparo V and Ramacciato G. Prognostic assessment of different lymph node staging methods for pancreatic cancer with $\mathrm{R} 0$ resection: $\mathrm{pN}$ staging, lymph node ratio, log odds of positive lymph nodes. Pancreatology. 2014; 14:289-294.

11. Ozawa T, Ishihara S, Sunami E, Kitayama J and Watanabe T. Log odds of positive lymph nodes as a prognostic indicator in stage IV colorectal cancer patients undergoing curative resection. J Surg Oncol. 2015; 111:465-471.

12. Arslan NC, Sokmen S, Canda AE, Terzi C and Sarioglu S. The prognostic impact of the log odds of positive lymph nodes in colon cancer. Colorectal Dis. 2014; 16:O386-O392.

13. Vinh-Hung V, Verschraegen C, Promish DI, Cserni G, Van de Steene J, Tai P, Vlastos G, Voordeckers M, Storme $\mathrm{G}$ and Royce M. Ratios of involved nodes in early breast cancer. Breast Cancer Res. 2004; 6:R680-R688.

14. Chen LJ, Chung KP, Chang YJ and Chang YJ. Ratio and log odds of positive lymph nodes in breast cancer patients with mastectomy. Surg Oncol. 2015; 24:239-247.

15. Touijer K and Scardino PT. Nomograms for staging, prognosis, and predicting treatment outcomes. Cancer-Am Cancer Soc. 2009; 115:3107-3111.

16. Sternberg CN. Are nomograms better than currently available stage groupings for bladder cancer? J Clin Oncol. 2006; 24:3819-3820.

17. Ichikura T, Ogawa T, Chochi K, Kawabata T, Sugasawa H and Mochizuki H. Minimum number of lymph nodes that should be examined for the International Union Against Cancer/American Joint Committee on Cancer TNM classification of gastric carcinoma. World J Surg. 2003; 27:330-333.

18. Lee HK, Yang HK, Kim WH, Lee KU, Choe KJ and Kim JP. Influence of the number of lymph nodes examined on staging of gastric cancer. Br J Surg. 2001; 88:1408-1412.
19. Kong SH, Lee HJ, Ahn HS, Kim JW, Kim WH, Lee $\mathrm{KU}$ and Yang HK. Stage migration effect on survival in gastric cancer surgery with extended lymphadenectomy: the reappraisal of positive lymph node ratio as a proper N-staging. Ann Surg. 2012; 255:50-58.

20. Marchet A, Mocellin S, Ambrosi A, Morgagni P, Garcea D, Marrelli D, Roviello F, de Manzoni G, Minicozzi A, Natalini G, De Santis F, Baiocchi L, Coniglio A and Nitti D. The ratio between metastatic and examined lymph nodes ( $\mathrm{N}$ ratio) is an independent prognostic factor in gastric cancer regardless of the type of lymphadenectomy: results from an Italian multicentric study in 1853 patients. Ann Surg. 2007; 245:543-552.

21. Wu SG, Wang Y, Zhou J, Sun JY, Li FY, Lin HX and He ZY. Number of negative lymph nodes should be considered for incorporation into staging for breast cancer. Am J Cancer Res. 2015; 5:844-853.

22. Kuru B. Prognostic significance of total number of nodes removed, negative nodes removed, and ratio of positive nodes to removed nodes in node positive breast carcinoma. Eur J Surg Oncol. 2006; 32:1082-1088.

23. Bochner BH, Kattan MW and Vora KC. Postoperative nomogram predicting risk of recurrence after radical cystectomy for bladder cancer. J Clin Oncol. 2006; 24:3967-3972.

24. Karakiewicz PI, Briganti A, Chun FK, Trinh QD, Perrotte P, Ficarra V, Cindolo L, De la Taille A, Tostain J, Mulders PF, Salomon L, Zigeuner R, Prayer-Galetti T, Chautard D, Valeri A and Lechevallier E, et al. Multi-institutional validation of a new renal cancer-specific survival nomogram. J Clin Oncol. 2007; 25:1316-1322.

25. Wierda WG, O'Brien S, Wang X, Faderl S, Ferrajoli A, Do KA, Cortes J, Thomas D, Garcia-Manero G, Koller C, Beran M, Giles F, Ravandi F, Lerner S, Kantarjian H and Keating M. Prognostic nomogram and index for overall survival in previously untreated patients with chronic lymphocytic leukemia. Blood. 2007; 109:4679-4685.

26. Wang J, Hassett JM, Dayton MT and Kulaylat MN. The prognostic superiority of $\log$ odds of positive lymph nodes in stage III colon cancer. J Gastrointest Surg. 2008; 12:1790-1796.

27. Harrell FJ, Lee KL and Mark DB. Multivariable prognostic models: issues in developing models, evaluating assumptions and adequacy, and measuring and reducing errors. Stat Med. 1996; 15:361-387. 